\title{
Nachhaltigkeit als Kriterium für Konsumentscheidungen
}

\author{
Nachhaltigkeitsaspekte gewinnen bei Konsument- \\ scheidungen für die Verbraucher an Bedeutung. \\ Auf Basis breit angelegter repräsentativer Um- \\ fragedaten wird analysiert, wie der Begriff in der \\ Bevölkerung rezipiert wird und inwiefern Verbrau- \\ cher in verschiedenen Konsumbereichen auf \\ Nachhaltigkeit bei ihren Kaufentscheidungen \\ achten. Von Oliver Bruttel
}

E s war ein langer Prozess, bis sich die Bevölkerung mit dem Begriff der Nachhaltigkeit vertraut gemacht hat. Noch im Jahr 2007 hatten erst 67 Prozent den Begriff „Nachhaltigkeit“ überhaupt schon einmal gehört, 2012 waren es bereits 81 Prozent, wie bevölkerungsrepräsentative Umfragen des Instituts für Demoskopie Allensbach aus diesen Jahren zeigen. Gestiegen ist auch der Anteil derjenigen, die auf Nachfrage zutreffend beschreiben können, was mit Nachhaltigkeit gemeint ist. 2007 war erst gut ein Drittel der Bürger in der Lage, spontan zutreffend zu sagen, was sie unter Nachhaltigkeit verstehen. Die anderen hatten von dem Begriff noch nicht gehört oder machten auf die offene Nachfrage keine oder falsche Angaben. 2012 waren es immerhin 55 Prozent, die „Nachhaltigkeit“ inhaltlich zutreffend beschreiben können. Das bedeutet aber auch, dass nach wie vor fast jeder zweite Bürger mit dem Begriff nicht viel anfangen kann.

\section{Karriere eines Begriffs}

Dabei gibt es erwartungsgemäß große Unterschiede, abhängig von der Schulbildung der Befragten. Von den Personen mit einfacher Schulbildung, also höchstens einem Hauptschulabschluss, haben erst 34 Prozent von dem Begriff gehört und können ihn inhaltlich zutreffend beschreiben; in der mittleren Bildungsschicht sind es 60 Prozent. Bemerkenswerterweise können aber auch in den höheren Bildungsschichten, also Personen, die mindestens über die Fachhochschulreife verfügen, nur drei Viertel den Begriff zutreffend umschreiben.

Von denjenigen, die den Begriff bereits gehört haben, umschreiben 26 Prozent diesen spontan mit eigenen Worten und ohne die Vorlage von Antwortalternativen ganz pauschal mit einer längerfristigen Perspektive und anhaltenden Wirkung. 17 Prozent beschreiben ihn mit einem sparsamen Umgang mit natürlichen Ressourcen, jeweils neun Prozent mit dem Den- ken an nachfolgende Generationen und Umweltschutz. Acht Prozent bringen Nachhaltigkeit mit einer verantwortungsvollen Zukunftsplanung in Verbindung, fünf Prozent sehen darin ganz allgemein ein positives, verantwortungsbewusstes Handeln. Rund ein Drittel derjenigen, die bereits von Nachhaltigkeit gehört haben, sind allerdings nicht in der Lage, den Begriff ungestützt mit Inhalt zu füllen.

Während die offene Abfrage ohne Antwortvorgaben gezeigt hat, dass ein Teil der Bevölkerung, obwohl sie den Begriff „Nachhaltigkeit“ bereits gehört hat, diesen inhaltlich nicht füllen kann, ordnen die Bürger gestützt, also bei Vorlage einer Liste mit möglichen Antwortalternativen, Nachhaltigkeit vor allem drei Bereichen zu:

I der Berücksichtigung der Interessen künftiger Generationen,

- Umwelt- und Klimaschutz,

- Fair Trade und Entwicklungshilfe.

Die Zuordnung wurde dabei auf Basis einer Faktorenanalyse vorgenommen. Eine Faktorenanalyse ist ein mathematischstatistisches Verfahren, mit dem festgestellt werden kann, welche Aussagen von den Befragten oft gemeinsam ausgewählt werden. Das Verständnis, das die Bevölkerung vom Begriff „Nachhaltigkeit“ hat, spiegelt dabei grob die Dimensionen des sogenannten Drei-Säulen-Modells der Nachhaltigkeit wider (Deutscher Bundestag 1998). Da ist zum einen die ökonomische Nachhaltigkeit, die die Interessen künftiger Generationen berücksichtigt und wirtschaftlich nicht über ihre Verhältnisse lebt. 62 Prozent der Bevölkerung verstehen unter Nachhaltigkeit, dass die Politik bei ihren Entscheidungen nicht nur an die heutige Generation, sondern auch an künftige Generationen denkt. 56 Prozent der Bevölkerung sehen darin auch eine persönliche Komponente, indem man bei langfristigen Entscheidungen auch an die Folgen für seine Kinder und Enkelkinder denkt.

Zum anderen wird von der Bevölkerung besonders die ökologische Dimension betont. Jeweils zwischen 50 und 60 Prozent verstehen unter Nachhaltigkeit den sparsamen Umgang mit Rohstoffen und Energie, den Schutz der Umwelt, die Wiederverwertung von Wertstoffen sowie die Nutzung erneuerbarer Energien.

Weniger häufig wird die soziale Dimension von der Bevölkerung unter Nachhaltigkeit subsumiert. Nur 36 Prozent der Bevölkerung verstehen unter Nachhaltigkeit, beim Kauf von Produkten darauf $\mathrm{zu}$ achten, dass diese unter gerechten Arbeitsbedingungen hergestellt wurden. 28 Prozent verbinden mit Nachhaltigkeit, dass man ärmere Länder dabei unterstützt, ihren Lebensstandard zu verbessern (siehe Abbildung 1). 


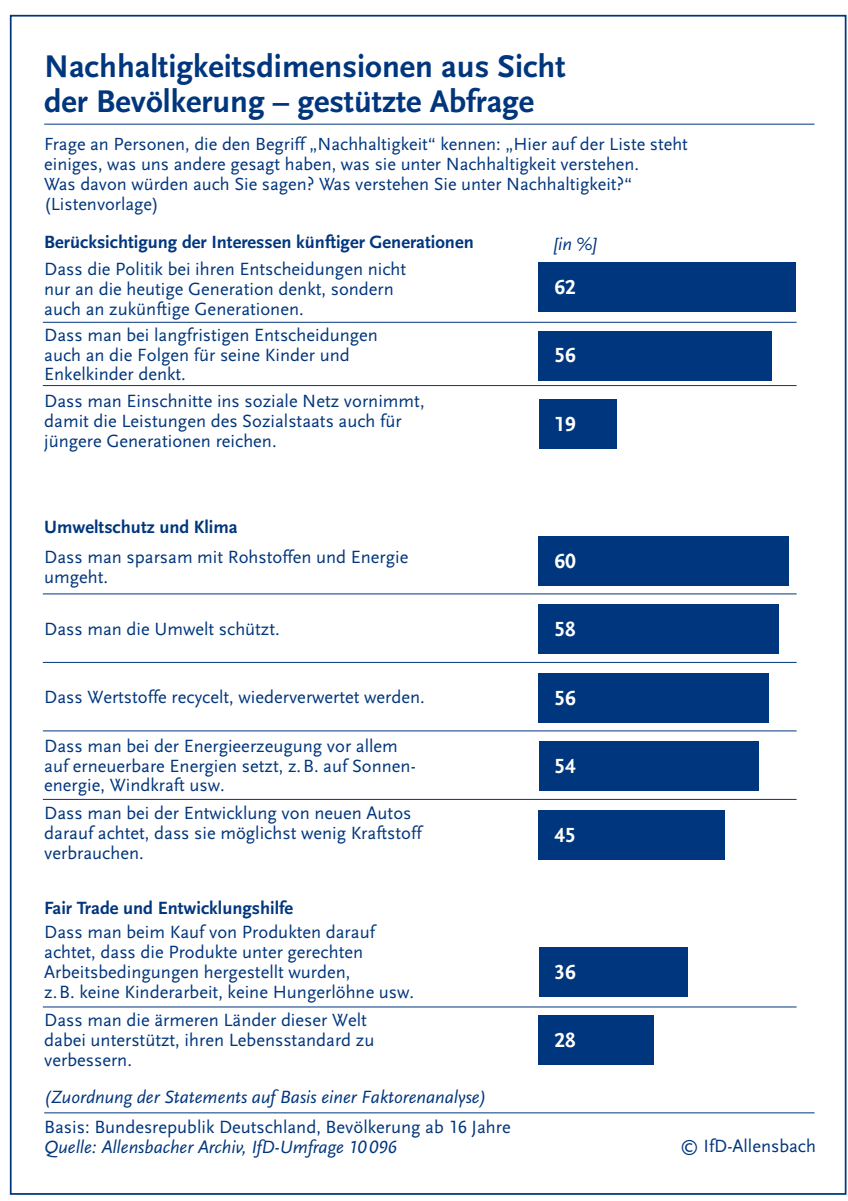

Abbildung 1: Nachhaltigkeitsdimensionen aus Sicht der Bevölkerung.

\section{Nachhaltigkeit als Konsumkriterium}

Angesichts der Vielschichtigkeit des Begriffs Nachhaltigkeit, sowohl im theoretischen Diskurs wie auch im Verständnis der Bevölkerung, bedarf es im Rahmen einer empirischen Studie, die die Relevanz von Nachhaltigkeit in verschiedenen Konsumbereichen untersucht, der Konkretisierung. Dies ist im Rahmen der Allensbacher Markt- und Werbeträgeranalyse, kurz auch als AWA bezeichnet, geschehen. Die AWA erscheint jährlich und stützt sich jeweils auf bundesweit mehr als 25.000 Face-to-Face-Interviews. Die Ergebnisse der AWA sind repräsentativ für die deutschsprachige Wohnbevölkerung ab 14 Jahre (Institut für Demoskopie Allensbach 2013).

Eine erste Übersicht belegt, dass die verschiedenen Dimensionen von Nachhaltigkeit, auch zwischen Konsumbereichen, für das Verbraucherverhalten einen unterschiedlichen Stellenwert haben. So schränken sich unabhängig von konkreten Lebens- beziehungsweise Konsumbereichen 31 Prozent der Bevölkerung, was 21,8 Millionen Verbrauchern ab 14 Jahren entspricht, bewusst ein, um die Umwelt zu schützen; zum Beispiel indem sie Wasser oder Strom sparen oder weniger Auto fahren (siehe Abbildung 2). Die wenigsten Verbraucher dürften aber die gesamte Wertschöpfungskette im Blick haben, wenn es um die Schonung natürlicher Ressourcen geht. So stellt der Wasserverbrauch durch den Endverbraucher natürlich nur einen Bruchteil des individuellen „Water Footprint“ dar (Sonnenberg et al. 2009). Gleiches gilt für den „Carbon Footprint“ bei der Mobilität jenseits des Automobils, beispielsweise bei Flugreisen.

Auch die Reduzierung von Verpackungsmüll ist jedem vierten Bürger, nicht zuletzt durch die vielen gesetzlichen Regelungen für den Einzelhandel wie die Rücknahme von Verpackungen im Laden, ein Anliegen. 17 Prozent der Bevölkerung achten bei Produkten auf Fair Trade, 15 Prozent auf das soziale und ökologische Verantwortungsbewusstsein von Unternehmen, also auf Corporate Social Responsibility (CSR).

Die beiden letztgenannten Kriterien, Fair Trade und CSR, haben sich dabei in den letzten Jahren unterschiedlich entwickelt. So ist die Bedeutung von Fair Trade kontinuierlich gestiegen. Gaben 2009 erst 13 Prozent der Verbraucher an, dass sie beim Kauf von Produkten darauf achten, dass diese aus fairem Handel stammen, sind es inzwischen 17 Prozent. Dies entspricht einem Plus von 31 Prozent seit 2009. Dies spiegelt sich auch in den deutlich gestiegenen Umsätzen mit Fair-TradeProdukten in Deutschland wider. Dagegen ist die Bedeutung von CSR als Entscheidungskriterium für die Verbraucher nicht gestiegen. Seit 2008 liegt der Anteil derjenigen, die beim Kauf von Produkten auf das soziale und ökologische Engagement der hinter dem Produkt stehenden Unternehmen achten, stabil zwischen 14 und 15 Prozent.

Im Vergleich verschiedener Konsumbereiche wie Lebensmittel, Automobil, Kosmetik, Kleidung und Reisen spielt Nachhaltigkeit für die Konsumentscheidung im Lebensmittelbereich die größte Rolle, nicht zuletzt, weil der „Bio“-Gedanke historisch dort besonders früh eine nennenswerte Verbreitung und Sichtbarkeit erlangt hat. 29 Prozent geben zu Protokoll, beim Kauf von Lebensmitteln besonders darauf zu achten, dass Produkte aus artgerechter Tierhaltung stammen, was je nach Verständnis nicht zwingend auch kontrolliert biologisch bedeuten muss. 23 Prozent der Bevölkerung legen generell Wert auf Bio-Produkte.

Bei Autos achten 20 Prozent darauf, ein möglichst umweltfreundliches Fahrzeug zu kaufen. Bei Kosmetika ist es 15 Prozent wichtig, dass diese nur aus natürlichen Inhaltsstoffen bestehen. Die umwelt- und sozialverträgliche Herstellung von Kleidung ist für zwölf Prozent der Verbraucher ein wichtiges Kaufkriterium. Noch am geringsten ausprägt ist der Nachhaltigkeitsgedanke bei den Verbrauchern im Bereich Tourismus.

\section{Der nachhaltige Verbraucher}

Um das Profil von Verbrauchern herauszuarbeiten, die besonders auf Nachhaltigkeit bei ihrem Verhalten sowie ihren Konsumentscheidungen achten, bietet es sich an, die genannten elf Kriterien mithilfe einer Skala zusammenzufassen. Definiert man „nachhaltige Verbraucher“ als die Personen, für 
die mindestens fünf der elf Dimensionen wichtig sind, repräsentieren diese eine Zielgruppe von 19 Prozent der Bevölkerung. Je nach Abgrenzung kann diese Gruppe auch größer oder kleiner sein. Würde man den Kreis so definieren, dass mindestens vier der elf Dimensionen genannt worden sein müssen, würden darunter 25 Prozent der Verbraucher fallen, bei mindestens sieben Statements würde der Kreis nur noch zehn Prozent der Verbraucher umfassen. Die damit umgrenzte Zielgruppe ist nur teilweise deckungsgleich mit der Gruppe der LOHAS (Lifestyles of Health and Sustainability), da insbesondere die Aspekte des Gesundheitsbewusstseins sowie der Naturverbundenheit als Abgrenzungskriterien hier nicht berücksichtigt wurden (vgl. Kirig/Rauch/Wenzel 2007).

Diejenigen, die in verschiedenen Bereichen auf Nachhaltigkeit bei ihrem Verhalten und ihren Kaufentscheidungen achten, weisen an vielen Stellen ein spezifisches Profil hinsichtlich ihrer soziodemografischen Merkmale, aber auch ihrer Wertvorstellungen auf. Die nachfolgenden Zahlen beziehen sich jeweils auf die Gruppe, die bei mindestens fünf der elf Dimensionen auf Nachhaltigkeit achtet. Konsumenten, die entsprechend dieser Definition auf Nachhaltigkeit achten, kommen überdurchschnittlich häufig aus den oberen sozialen Schichten, verfügen also über ein überdurchschnittliches Einkommen und mehr noch über eine überdurchschnittliche Bildung. Frauen gehören häufiger als Männer zur Gruppe der nachhaltigen Konsumenten, was teilweise auch daran liegt, dass sie grundsätzlich in vielen Bereichen nach wie vor deutlich häufiger für den Einkauf zuständig beziehungsweise konsumfreudiger sind. Besonders ausgeprägt ist der Nachhaltigkeitsgedanke zudem bei den Älteren (siehe Abbildung 3). Aus den vorgenannten Ergebnissen lässt sich eine Gruppe ableiten, in der nachhaltiger Konsum besonders ausgeprägt ist. Dies sind ältere Frauen aus den oberen sozialen Schichten. Von den 60-jährigen und älteren Frauen aus den oberen sozialen Schichten achten 44 Prozent besonders stark auf Nachhaltigkeit bei ihren Konsumentscheidungen. Dies sind deutlich mehr als in allen anderen Schichten, insbesondere auch in den gebildeten jüngeren und mittleren Altersgruppen. Familien mit Kindern unter sechs Jahren achten in Summe interessanterweise eher unterdurchschnittlich auf Nachhaltigkeit, wenngleich es natürlich Bereiche wie Bio-Lebensmittel gibt, bei denen die Nachhaltigkeitsorientierung von Familien mit kleinen Kindern besonders stark ausgeprägt ist.

Die Wertvorstellungen der nachhaltigen Verbraucher sind überdurchschnittlich stark geprägt von Verantwortungsbewusstsein, sozialer Gerechtigkeit und politischer Teilhabe, aber auch von Naturverbundenheit, Kreativität und der Auseinandersetzung mit den Sinnfragen des Lebens. Dagegen ist ihnen das Streben nach einem hohen Einkommen und materiellem Wohlstand weit weniger wichtig. Am sympathischsten sind ihnen mit jeweils 27 Prozent Bündnis 90/Die Grünen und die CDU/CSU.

Die Interpretation von nachhaltigen Verbrauchern als eine homogene Zielgruppe ist auf der einen Seite hilfreich. Auf der

\section{Relevanz von Nachhaltigkeit für die Konsum- entscheidung}

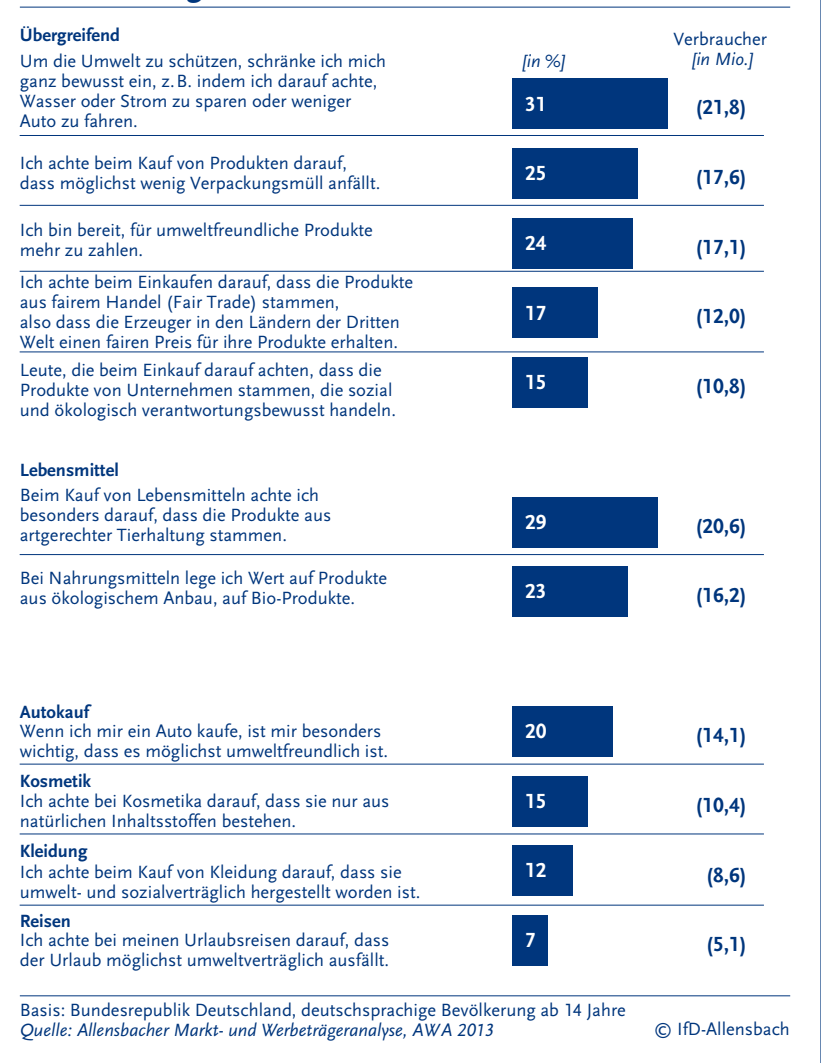

Abbildung 2: Relevanz von Nachhaltigkeit für die Konsumentscheidung.

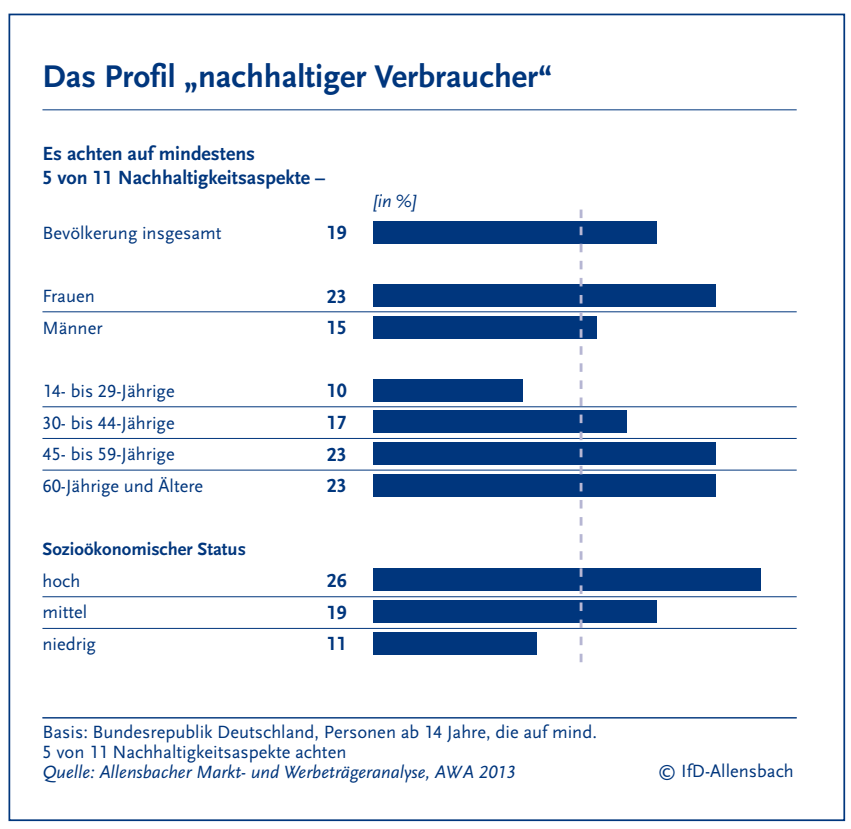

Abbildung 3: Das Profil „nachhaltiger Verbraucher“. 
anderen Seite besteht aber die Gefahr, dass dabei übersehen wird, dass der Stellenwert verschiedener Nachhaltigkeitsdimensionen bei Konsumentscheidungen auch innerhalb dieser Zielgruppe teilweise individuell sehr unterschiedlich ist. So legt zum Beispiel von denjenigen, die beim Lebensmittelkauf auf Bio-Produkte achten, nur jeweils rund ein Drittel auch auf natürliche Inhaltsstoffe bei Kosmetika oder die Umwelt- und Sozialverträglichkeit bei Kleidung Wert. Für jeweils weniger als die Hälfte der Bio-Käufer im Lebensmittelbereich ist Fair Trade oder die Umweltfreundlichkeit beim Autokauf ein besonderes Anliegen (siehe Tabelle 1). Daher ist es aufschlussreich, die einzelnen Konsumbereiche im Detail zu beleuchten.

\section{Nachhaltigkeit bei Konsumentscheidungen in verschiedenen Bereichen}

Der Anteil der Bevölkerung, der zu Bio-Produkten greift, ist in den letzten drei Jahrzehnten stetig gestiegen. 1985 haben nur fünf Prozent der westdeutschen Bevölkerung biologisch angebautes Obst, Gemüse oder Salat gekauft (bezogen auf den Zeitraum 14 Tage vor Durchführung der Befragung). Bis zum Jahr 2000 ist dieser Anteil auf 15 Prozent gestiegen, 2005 lag der bei 18 Prozent. Anschließend kam ein deutlicher Anstieg: 2010 griffen 30 Prozent der westdeutschen zu frischen Bio-Produkten; derzeit sind es 31 Prozent. „Bio“ hat sich damit von einer Nische zu einem Massenmarkt entwickelt. Aber nach wie vor sind es vor allem, gemessen an Einkommen und Bildung, die oberen sozialen Schichten, mit wenigen Abstrichen auch die mittleren und nicht zuletzt Familien mit kleinen Kindern, die besonders häufig zu Bio-Produkten greifen.

Die Lebensmittelskandale haben das Vertrauen der Bevölkerung in die Qualität der Lebensmittel in Deutschland dabei kaum erschüttert. Zwischen 2006 und 2012 schwankte der Anteil derjenigen, die die Qualität der Lebensmittel in Deutschland als „sehr gut“ oder "gut“ einschätzten, zwischen 71 und
86 Prozent. Nur zwischen elf und 25 Prozent stuften die Lebensmittelqualität als „weniger gut“ oder „gar nicht gut“ ein.

Um ein genaueres Verständnis über Verbraucher zu erhalten, denen es beim Autokauf besonders wichtig ist, dass das Auto möglichst umweltfreundlich ist, ist es sinnvoll, diejenigen genauer zu analysieren, die in den kommenden ein bis zwei Jahren den Kauf eines Pkw planen. Denn nur für diese bietet sich die Möglichkeit, ihre Nachhaltigkeitspräferenz beim Automobilkauf auch konkret umzusetzen.

Legt man diesem Personenkreis eine Liste mit Merkmalen für die Wunschausstattung ihres Autos vor, dann lässt sich erkennen, was für sie ein umweltfreundliches Auto auszeichnet. Von denjenigen, die beim Autokauf auf ein möglichst umweltfreundliches Auto achten, zählen 13 Prozent einen Hybridantrieb zu ihrer Wunschausstattung. Dies sind fast doppelt so viele wie der Durchschnitt der Pkw-Käufer. Deutlich unterdurchschnittlich ist dagegen der Wunsch nach einer hohen PS-Zahl, Sportausstattung oder Breitbereifung ausgeprägt. In der überwiegenden Zahl der anderen Kriterien unterscheiden sich umweltbewusste Autokäufer allerdings kaum vom Durchschnitt der Autokäufer.

Vor allem Kosmetika, teilweise aber auch Kleidung, sind Bereiche, in denen es vor allem Frauen sind, die auf Nachhaltigkeit achten. Das liegt nicht zuletzt darin begründet, dass Frauen gerade im Bereich Kosmetik mehr Produkte nutzen als Männer. So achten 24 Prozent der Frauen, aber nur fünf Prozent der Männer auf rein natürliche Inhaltsstoffe bei Kosmetika. Beim Kleidungskauf achten 15 Prozent der Frauen und neun Prozent der Männer auf Nachhaltigkeit. Mit Blick auf die Altersstruktur fällt ins Auge, dass es keinen anderen Konsumbereich gibt, in dem die 14- bis 29-Jährigen im Vergleich zum Bevölkerungsdurchschnitt derart wenig Wert auf Nachhaltigkeit legen wie im Modebereich, der von den Jüngeren für sich selbst gleichzeitig als besonders wichtig eingestuft wird. Gerade einmal fünf Prozent der 14- bis 29-Jährigen achten beim Kauf von Kleidung auf

\begin{tabular}{|c|c|c|c|c|c|c|c|}
\hline & \multicolumn{7}{|c|}{ Personen, die beim Einkauf auf... achten, - } \\
\hline & CSR & Fair Trade & $\begin{array}{l}\text { Bio-Lebens- } \\
\text { mittel }\end{array}$ & $\begin{array}{l}\text { Umweltfreund- } \\
\text { lichkeit beim } \\
\text { Autokauf }\end{array}$ & $\begin{array}{l}\text { Natürliche } \\
\text { Inhaltsstoffe } \\
\text { bei Kosmetika }\end{array}$ & $\begin{array}{l}\text { Umwelt- und sozial- } \\
\text { verträgliche Herstel- } \\
\text { lung von Kleidung }\end{array}$ & $\begin{array}{l}\text { Umwelt- } \\
\text { verträglichkeit von } \\
\text { Urlaubsreisen }\end{array}$ \\
\hline - achten auch auf: & $\%$ & $\%$ & $\%$ & $\%$ & $\%$ & $\%$ & $\%$ \\
\hline CSR & 100 & 49 & 45 & 34 & 38 & 55 & 48 \\
\hline Fair Trade & 54 & 100 & 49 & 38 & 44 & 59 & 53 \\
\hline Bio-Lebensmittel & 67 & 66 & 100 & 45 & 55 & 61 & 59 \\
\hline $\begin{array}{l}\text { Umweltfreundlichkeit } \\
\text { beim Autokauf }\end{array}$ & 44 & 45 & 39 & 100 & 37 & 47 & 57 \\
\hline $\begin{array}{l}\text { Natürliche Inhaltsstoffe } \\
\text { bei Kosmetika }\end{array}$ & 36 & 38 & 35 & 28 & 100 & 38 & 40 \\
\hline $\begin{array}{l}\text { Umwelt- und sozialverträgliche } \\
\text { Herstellung von Kleidung }\end{array}$ & 44 & 42 & 32 & 29 & 31 & 100 & 47 \\
\hline $\begin{array}{l}\text { Umweltverträglichkeit } \\
\text { von Urlaubsreisen }\end{array}$ & 23 & 22 & 19 & 20 & 20 & 28 & 100 \\
\hline
\end{tabular}

Basis: Deutschsprachige Bevölkerung ab 14 Jahre

Tabelle 1: Unterschiedliche Relevanz des Nachhaltigkeitsgedankens für individuelle Verbraucher über verschiedene Konsumbereiche hinweg. 
deren umwelt- und sozialverträgliche Herstellung; im Durchschnitt der Bevölkerung sind es zwölf Prozent.

Frauen, die auf Nachhaltigkeit im Bekleidungsbereich achten, sind weder in der jüngeren Generation noch in den anderen Altersgruppen Modemuffel. Vielmehr interessieren sich diejenigen Frauen, die im Bekleidungsbereich auf Nachhaltigkeit achten, kaum weniger für Mode und Modetrends und legen praktisch genauso viel Wert darauf, gut gekleidet zu sein, wie Frauen insgesamt. Auch in puncto Markenbewusstsein unterscheiden sich die beiden Gruppen kaum.

Im Vergleich $\mathrm{zu}$ den anderen Konsumbereichen spielt Nachhaltigkeit im Reisebereich derzeit eine deutlich untergeordnete Rolle. Die Gruppe derjenigen, die beim Reisen besonders auf die Umweltverträglichkeit achten, unterscheidet sich hinsichtlich bevorzugter Urlaubsart und Urlaubszielen allerdings teilweise deutlich von der Gesamtbevölkerung. Um andere Einflussfaktoren wie Alter und Einkommen zu kontrollieren, bietet sich eine Analyse der 20- bis 59-Jährigen mit einem monatlichen Haushaltsnettoeinkommen von 3.000 Euro und mehr an. Nimmt man diese Gruppe als Basis und vergleicht dabei die Gruppe insgesamt mit denjenigen in dieser Gruppe, die auf die Umweltverträglichkeit ihrer Reisen achten, so fällt zum einen die ausgeprägte Vorliebe der „nachhaltigen Reisenden“ für Wander- beziehungsweise Rucksackurlaube, Kulturreisen, Sprachurlaube, Urlaub auf dem Bauernhof oder auf dem Land sowie andere Aktiv- oder Sporturlaube auf, während sie sich gleichzeitig deutlich weniger für Cluburlaube und Kreuzfahrten erwärmen können. Zum anderen machen sie überdurchschnittlich häufig Urlaub in Deutschland. 30 Prozent der 20bis 59-Jährigen mit einem monatlichen Haushaltseinkommen von 3.000 Euro und mehr haben in den letzten zwölf Monaten Urlaub in Deutschland gemacht; von denjenigen, die in dieser Zielgruppe besonders auf die Nachhaltigkeit ihres Urlaubs Wert legen, waren es 46 Prozent.

\section{Fazit}

Nachhaltigkeit hat für die Bevölkerung viele Facetten. Insofern ist es wichtig, diese in Diskussionen und wissenschaftlichen Arbeiten möglichst präzise zu fassen. Die Verbraucher sind durchaus bereit, Nachhaltigkeit in ihre Konsumentscheidungen mit einfließen zu lassen. Bisher ist Nachhaltigkeit aber nur im Lebensmittel- und Automobilbereich für einen nennenswerten Teil der Bevölkerung ein Entscheidungskriterium. Bei Kleidung, Kosmetik und Reisen ist der Nachhaltigkeitsgedanke noch nicht sonderlich verbreitet. Allerdings hat das Beispiel „Bio“-Lebensmittel gezeigt, wie rasch ein Leitbild von einer relativ kleinen Zielgruppe bei entsprechend günstigen Rahmenbedingungen Relevanz für breitere Schichten der Bevölkerung entwickeln kann.

\section{Literatur}

Deutscher Bundestag (1998): Abschlussbericht der Enquete-Kommission „Schutz des Menschen und der Umwelt - Ziele und Rahmenbedingungen einer nachhaltig zukunftsverträglichen Entwicklung“.

Institut für Demoskopie Allensbach (2013): Allensbacher Markt- und Werbeträgeranalyse (AWA). Im Internet unter: www.ifd-allensbach.de/awa.

Kirig, A./Rauch, C./Wenzel, E. (2007): Zielgruppe LOHAS - Wie der grüne Lifestyle die Märkte erobert. Kelkheim.

Sonnenberg, A./Chapagain, A./Geiger, M./August, D. (2009): Der WasserFußabdruck Deutschlands: Woher stammt das Wasser, das in unseren Lebensmitteln steckt? WWF Deutschland, Frankfurt.

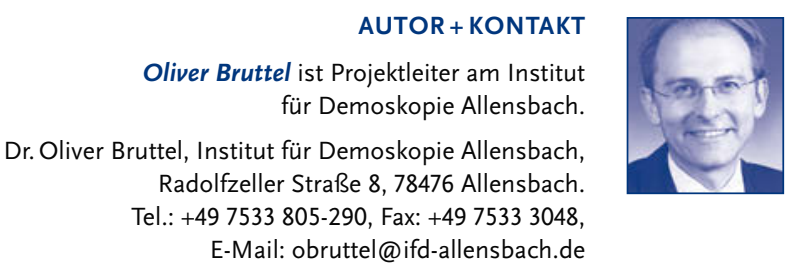

\section{$\mathrm{K}$ wie Kooperation}

Die Geschichte unserer Zivilisation ist voller Beispiele von Kulturen, die aufgrund der Anpassung an ihre natürliche Umgebung unter widrigsten Umständen bestehen konnten - oder an der Ausbeutung ihrer natürlichen Ressourcen zugrunde gingen. Diese Spurensuche zur Geschichte der Nachhaltigkeit berichtet vom Umgang mit Bevölkerungswachstum, Umweltverschmutzung und Naturkatastrophen und hilft uns, die richtigen Lehren daraus zu ziehen.

U. Schneckener, A. von Scheliha, A. Lienkamp, B. Klagge (Hrsg.)

Wettstreit um Ressourcen

Konflikte um Klima, Wasser und Boden

282 Seiten, broschiert, 29,95 Euro, ISBN 978-3-86581-421-0

Erhältlich bei

www.oekom.de | oekom@verlegerdienst.de

Die guten Seiten der Zukunft 